\title{
Approaching Mental Disorders from the Engineering Point of View
}

\author{
J. Aguiló \\ Department of Microelectronics and Electronic Systems, Universidad Autónoma de Barcelona, Barcelona, \\ Spain \\ Biomedical Research Networking Center (CIBER-BBN), Spain \\ acmlis.conference@gmail.com
}

\begin{abstract}
Mental illness and mental disorders represent an increasing burden affectingthepopulation of all ages at all places, challenging mental health and health systems and contributing to the onset or to the acceleration of many other diseases. Cardiovascular risk, diabetes or also depression among many others seems to be clear examples of these effects. Concerning depression, there is a critical cross feedback: Stress causes depression whilethat staying in depressive states causes stress.

An accurate, reliable and almost continuous monitoring of the "instantaneous" state of the subjects would undoubtedly contribute to a better diagnostic and follow-up. So, treatments would be personalized by fine tuning relationships between drugs or other kind of interventions and subject state which in turn would contribute to a better knowledge of the causes of the disease and of the mechanisms acting in its treatment.

Following this primary thought,a research line explicitly focusing quantitative assessment methods and related technologies was stablished at the Universidad Autónoma de Barcelona.

First results had come from the study of moderate stress. In collaboration with various technological and clinical groups we proposed a reference scale to measure the stress level and we have taken the first steps concerning its validation; we are proposing a set of weighed parameters coming from physiological signals as a multivariable biomarker including some unexpected components. This "biomarker" show a strong correlation with the reference scale then stress can be quantitatively assessed continuously, reliably, repeatable and avoiding most of the subjective components of the patient and the observer.From these results, it is expected to demonstrate that a tool is available to determine, for example, the quality of an empowerment session, the effectiveness of an intervention using mindfulness in children with TDAH, or to determine the extent to which biofeedback techniques are useful as tools to improve quality of life of primary caregivers of chronic patients.
\end{abstract}

Some of the pilots planned at the very beginning are still underway while new research in Depression, Epilepsy, MS and Alzheimer is under way following similar guidelines.

Keywords-Mental disorders, Mental Health, Reference Scale of severity, Multivariable biomarker 


\section{Introduction}

It is well known that from ancient Egypt, and probably before, the study of mental illness was of concern to society and to those in charge of science and health development, that even proposed and tried out surgical solutions to certain problems.

However, up to now advances in this area are not so fast than in most other medical fields. This is probably due to its inherent complexity and to the difficulties in finding atrue gold standard for testing methodologies allowing a reliable assessment.

Meanwhile, the numbers grow dramatically. The population in Europe is aging at an unprecedented proportion as the result of declining reproduction rates and increasing life expectancy. Because of that, chronic diseases are the main cause of illness in old age but mental disorders such as dementia and latelife depression play also a significant role as well as epilepsy and Alzheimer. Besides, because of the constant pressureof the modern way of life imposes on the individuals, stress is also dramatically growingup to point that the World Health Organization called it a World-Wide Epidemic.

In particular, the number of patients with dementia is expected to rise sharply as the prevalence of dementia doubles every 5.2 years exponentially between 65 and 85 years of age. Late-life depression is also an important public health problem. Estimated 1-year prevalence rates of depression range from $3 \%$ to $10 \%$. For dementia, prevalence rates are estimated between $0.6 \%$ and $3.7 \%$ for 65 -year-old to 69 -yearold, and $25.2 \%$ to $75 \%$ for adults 90 years old or older. Additionally, multiple studies have shown high comorbidity between mental disorders and chronic physical illnesses in the elderly. Positive feedback has also been demonstrated between different diseases such as diabetes mellitus, cancer and cardiovascular disease and the occurrence of depression in the elderly. And vice-versa, in older populations, there is also a positive feedback between depression and hypertension, diabetes and cardiovascular illnesses.

As it was pointedout by the Global Alliance for Chronic Diseases, "Mental disorders represent an everincreasing burden, to all ages of the population, challenging mental health and health systems. For example, depression affects 350 million people in communities across the world and represents the third leading contributor to the global disease burden. Dementia affects 47.5 million people worldwide with $58 \%$ of people living with dementia in low- and middle-income countries. Global costs associated with mental disorders were estimated to be $\$ 2.4$ trillion in 2010 and are expected to rise to $\$ 5.8$ trillion by 2030. Mental disorders cause tremendous suffering for individuals, families, communities and societies. They also increase the risk of co-morbid illnesses and social exclusion. There are obstacles to achieving effective prevention, early identification and management of mental disorders and to ensuring patients' adherence to therapies.

Coming from the Micro-Nano-Bio Systems design and prototyping to solve problems in graft transplant, in heart surgery, in functional recovery of ear and vision or in early detection of glaucoma, the GBIO research group from CIBER-BBN decided to explore possible technology help in monitoring mental health and wellbeing.

Two amazing things comes up from the first moment to the engineering-based team facing the quantitative assessment of emotional or psychological stress: the lack of a precise definition except in very severe manifestations and the lack of a reliable, totally objective and repeatable method of the severity measurement method. These two things were the first topics to afford. 
Although we are now working with different emphasis in Depression, TDAH, Alzheimer, Multiple Sclerosis and Epilepsy we start by trying to study stress. We thought that stress, when not severe or traumatic stress, were not a real illness so, in this way, we would not interfere with medical research. Besides, to implement results that we would have, if any, we will need to assume responsibility only concerning individual wellbeing of treated individuals, not concerning its health.

Once focus on moderate stress was decided, the main aim of the study would be summarized as attempting to quantify the level of stress as well as to find and to develop appropriated devices and/or methodologies to have an objective, reliable, and repetitive measurement procedure set-up.

Availability of such a tool will facilitate precise communications among professionals, allow a close followup of patients, will help doctors in diagnostic but also in the prescription of the medication and in quantifying the efficacy of the drugs. Establishment of thresholds will become also available in such a way that illness condition or not would be quantitatively established as well as related risks in some professional situations such as drivers, air controllers, police, firefighters, army, etc.

\section{Methodology and Experiment's Organization}

As already stated, the first step was to establish a definition of stress that would be acceptable from thepsychiatric point of view whilst clear from the engineering point of view. We took the following statement as stress definition: The "distance" measuringhow close or how far is the individual positioned from the medically and socially considered the "normal" status. This distance measured in a n-dimensional space where all the parameters related to stress should be considered. When considering acute stress this distance is considered between the everyday typical values of all considered variables, that is its point of homeostatic equilibrium, and the actual values of them after the stress stimulus. In chronic stress, the "normal" status should be taken among all the population with similar characteristics.

Concerning stress related information, first of all, we took as reference the psychometric test commonly used by professionals together with a commonly used and well documented moderate stressor. Stressor stimulus can be induced in the laboratory through a mental arithmetic task, public speaking task, by color word test, by hyperventilation, seeing film fragments, or using cold pressor test, among. Combining some of the already mentioned stressors, the Trier Social Stress Test (TSST) is so far one of the widely used and documented protocols in stress research [1], and it has been shown to exert major effects on almost all psycho-biological response mechanisms [2]. In a similar way, the autogenic relaxation method was used to induce a relaxed stated that will be used as the basal state of each individual.

On the other hand, the most frequently used psychometric test concerning stress are: the subject's selfreport assessments, the Visual Analogue Scale for Stress (VASS), the State-Trait Anxiety Inventory test (STAI-s). We also include some tests to evaluate individual trait against a stress stimulus. We take the (STAI-t) and the Perceived Stress Scale(PSS)

Researchers studying animal response to stress stimulus are usually using a set of specific biochemical markers to evaluate themagnitude of the response. Mainly they are using catecholamine, prolactin, copeptin, glucose, cortisol, $\alpha$-amylase and recently some inflammation markers such as interleukin- 6 and/or 10 or other cytokines as well as some tumor markers such as the TNF-a. Following this way, they have a more or less complete information about the endocrine and nervous responses of the organism to the stress stimulus. 
Nevertheless, biochemical markers are an invasive measure, expensive and its correct collection depends on the time gap since the stressor is active. On the other side, psychometric tests are very related with individual subjectivity and lose reliability when they are frequently applied.

It is clear, for example, that emotions, stress and some mental disorders are altering the cardiovascular function through complex interactions that include feedforward and feedback mechanisms involving effects of the autonomic nervous system (ANS) on it. Processing electrocardiography (ECG) signals to extract further information usually consists of analyzing time series representing the heart rate, intervals related to the activation and repolarization of the myocardium, systolic and diastolic blood pressure, and markers related to pulse wave velocity and peripheral resistance. Then respiration and chest movement, mechanical properties of the vasculature, such as the blood pressure and volumetric measures of the vessels seems to be direct or indirectly related to the stress related changes in the homeostatic equilibrium. A similar argument could be applied to electromyography (EMG) and also to skin temperature and skin conductance. All these signals could become key information sources that in fact are already used to evaluate stress effects in specific conditions.

Table 1. Experimental pilots set-up

\begin{tabular}{|c|c|c|}
\hline Type & $\begin{array}{l}\text { Title and short } \\
\text { description }\end{array}$ & $\begin{array}{c}\text { Responsible } \\
\text { Institution \&Place }\end{array}$ \\
\hline \multirow{3}{*}{ Psychological Stress } & $\begin{array}{c}\text { Relax vs. stress induced } \\
\text { by the TSST in Healthy } \\
\text { young subjects }\end{array}$ & $\begin{array}{l}\text { U. Aut. Barcelona } \\
\text { U. Poltech. Madrid } \\
\text { U. Zaragoza }\end{array}$ \\
\hline & $\begin{array}{c}\text { Parents of children } \\
\text { diagnosed with a severe } \\
\text { surgery }\end{array}$ & H. St Joan de Deu \\
\hline & $\begin{array}{l}\text { Patients diagnosed of } \\
\text { Depression syndrome }\end{array}$ & $\begin{array}{l}\text { H.C. Zaragoza } \\
\text { H. St Joan de Deu }\end{array}$ \\
\hline \multirow{2}{*}{ Chronic stress } & $\begin{array}{c}\text { Caregivers of chronic } \\
\text { patients }\end{array}$ & H. C. Barcelona \\
\hline & $\begin{array}{c}\text { Caregivers of terminal } \\
\text { oncologic }\end{array}$ & H. C. Barcelona \\
\hline \multirow{3}{*}{ Traumatic stress } & Knee arthroplasty & H. C. Barcelona \\
\hline & $\begin{array}{c}\text { scoliosis surgery in } \\
\text { children }\end{array}$ & H. St Joan de Deu \\
\hline & $\begin{array}{c}\text { Craniostenosis surgery } \\
\text { in children }\end{array}$ & H. St Joan de Deu \\
\hline Thermal stress & $\begin{array}{c}\text { Healthy young subjects } \\
6 \text { h. at } 35 \circ \text { oc, } 50 \% \\
\text { humidity }\end{array}$ & U. Aut. Barcelona \\
\hline $\begin{array}{c}\text { Asthma vs } \\
\text { Psychological stress }\end{array}$ & Asthma patients & H. St Pau, Barcelona \\
\hline $\begin{array}{l}\text { Effect of mindfulness } \\
\text { interventions in TDAH }\end{array}$ & $\begin{array}{c}\text { Children diagnosed of } \\
\text { TDAH syndrome }\end{array}$ & H. St Joan de Deu \\
\hline $\begin{array}{l}\text { The role of physical and } \\
\text { cognitive information } \\
\text { in } D C L\end{array}$ & $\begin{array}{l}\text { Recent diagnosed } \\
\text { Alzheimer patients }\end{array}$ & Fund. ACE Barcelona \\
\hline $\begin{array}{c}\text { Effect of biofeedback } \\
\text { interventions in } \\
\text { caregivers }\end{array}$ & $\begin{array}{l}\text { Caregivers of terminal } \\
\text { oncologic patients }\end{array}$ & H. C. Barcelona \\
\hline
\end{tabular}

In a mono-variate approach, these methods can be used to analyze the variability of single time series. In particular, the heart rate variability has been extensively studied because of its close interaction with the activity of the sympathetic and parasympathetic nervous systems. The spectral or time-frequency representation of the heart rate variability shows two main oscillations (called low frequency andhigh frequencycomponents) whose ratio can be used to monitor the sympathovagal balance. 
The multivariate analysis of the interactions between cardiovascular and cardiorespiratory signals gives further information about the ANS modulation. Novel frameworks have been recently proposed to describe the dynamic (causal) interactions between cardiovascular variables, which include the strength of the coupling, the direction of information flow and the latency in coupled changes. For example, mental stress and emotions have been shown to induce consistent pattern of responses in these interactions, which can be used to continuously monitoring the psychophysiological state in patient follow-up.

To cover the different types of stress, while attemptingto gain information that can be useful regardlessof the stressor, the methodis structured around nine initial pilot studies conducted indifferent samples of individuals that involve differenttypes of stress, as well as varying types and intensitiesof stressors or application times. Each of the pilotsis a prospective observational study with a specific protocol that wasapproved by the correspondingEthics Committee. A priori estimations indicatedthat a sample that could be used as a statistically significant indicator for each individual trial and thatwas powerful enough to contribute to thedevelopment of the joint algorithm must consist ofno fewer than $\mathbf{3 0}$ individuals. Forty volunteer subjectswere selected for each trial, all of whom fulfilledthe required eligibility criteria, in the hope that theminimum figure would be reached despite the dropoutsand those excluded due to errors [3].

Four additional experimental pilots have been added during the last two years due to the research interests of the hospital medical community.

Each trial consists of studying the action of aparticular stressor, which could be real or inducedartificially. In each of these sessions, which correspondto different states of the subject, the actionof the stressor upon the electrophysiological, biochemicaland psychometric variables established inthe corresponding protocol was evaluated while atthe same time the temporal sequences of their valueswere also collected [4].

In most of the trials the reference was taken as beingthe actual individual under conditions that couldbe considered his or her normal state. In the trials inwhich this is not possible, the reference used was asubject with the same characteristics, paired by age,sex, etc., but without the presence of the stressor.

The method implemented attempts to avoid theerrors and uncertainty due to the type or form ofmeasurement. Hence, homogeneous protocols weredesigned for the taking of measurements and processing of samples for the different trials. The electrophysiologicalmeasurements are performed usingthe same calibrated commercial equipment with CE certification following a single procedure for placingthe sensors and for recording. Each of the biochemicalvariables (analytes) are determined in the samespecialized laboratory that will process the samplesfrom all the pilots that include the valuation of this analyte. The same considerations were applied to the analyses to be carried out on saliva and hair.

The recent technological effort in the biomedical field is including the incorporation of sophisticated but affordable electronic sensors, but also means a dramatic increase in calculating capacity and thus the use of sophisticated algorithms allowing to extract more and more information from simple and apparently well know signals.

An example already well developed is the "fine" analysis of the ECG signal as mentioned above. The ECG signal is affected by the electronic noise of the environment, but also by breathing, movement or muscle contractions, speech and even by the ambient or body temperature among others. A careful selection of 
time windows with little interferencestogether with sophisticated signal processing algorithms to correct the aforementioned effects, is contributing to extract a greater amount of information from ECG and thus a greater and better knowledge of the behavior of the cardiovascular system but also of the whole organism including nervous system and brain functions. Our group recently contributed to this aspect by including the respiratory frequency information in the Heart Rate Variability, HRV, for a better analysis of Stress [5].

Following and extending this way, infrared cameras can be used in the near future, for the contact-less and remote record of the ECG and PPG signals, the skin temperature at different points and possibly the skin conductance. And from that the Pulse Transit Time, PTT, and the arterial pressure will be easily derived. And even more so, in a few years the voice signal recorded and processed from our own smartphone will be enough to predict a heart attack or an epilepsy seizure and warn the doctor and / or family if a memory failure that can cause problems is occurring.

Table 2. Set of parameters extracted from ECG, PPG and ST

\begin{tabular}{|c|c|c|}
\hline Indices from HRV & Indices from PPG & Indices from skin $T$ \\
\hline HRmean (bpm) & PRmean & \multirow{2}{*}{$\Delta T^{F I}$} \\
\hline SDNN (s) & $\mathrm{PP}_{\text {SDNN }}$ & \\
\hline RMSSD (s) & PPrMSSD & \multirow{2}{*}{$T_{f i}$ Ptotal } \\
\hline PPNN (\%) & $\mathrm{PDT}_{\text {mean }}$ & \\
\hline Power VLF (s-2) & PDT $_{\text {STD }}$ & \multirow{2}{*}{$\Delta T^{F a}$} \\
\hline Power LF(s-2) & PRTSTD & \\
\hline Power HF(s-2) & $\mathrm{PTT}_{\text {mean }}$ & \multirow{2}{*}{$T_{\text {fa }} a_{\text {Ptotal }}$} \\
\hline Power PLF(s-2) & $\mathrm{PTT}_{\mathrm{STD}}$ & \\
\hline Power PHF(s-2) & PWrstD & \multirow{2}{*}{$T_{\text {ratio }}$} \\
\hline Total power(s-2) & - & \\
\hline LF/Hf ratio & - & \\
\hline
\end{tabular}

Methods and algorithms to extract information from this sort of multimodal databaseare diverse too, from simple statistics to apply sophisticated classification techniques before using machine learning algorithms such as support vector machines, fuzzy logic, Bayesian networks, classification trees, artificial neural networks, Computer Aided Diagnostic tools orMulti-Task Gaussian processes.

Clearly, all these technological contributions are paving the road to a new paradigm in health care. The way in which data with diagnostic value are collected, analyzed and stored will change radically. The amount of available data will increase dramatically, offering the opportunity to evaluate diagnostic tools and treatments in a more accurate way and by using completely different equipmentSufficiently friendly and robust to be used mostly by the user itself.

\section{Why A Reference Scale?}

Measuring is a basic process carefully used in both science and engineering. It consists of comparing with a known pattern the phenomenon whose magnitude is desired to value. In many cases, mental health clinics lack this kind of "pattern", because psychometric test could be easily falsified by the patient and cannot be applied too frequently.

Nevertheless, measuring is crucial to communicate to others, to advance knowledge rapidly and to facilitate comparison. Medicine is no stranger to it and, with increasing force, tries to establish principles and establish solid foundations and links between diagnosis and follow-up based on objective measures. 
To measure it is necessary to have a reference: A comparison pattern allowing to quantitatively assess the magnitude to be measured. Conscious of the need, it was decided to take the pattern followed in 1985 at the George Washington University Hospital, Washington D.C., to score severity-of-disease of patients arriving at the Intensive Care Unit in such a way that higher scores correspond to more severe disease and a higher risk of death [6][7]. Physical, Psychological and Cognitive aspects were taken into account by using psychometric test and biochemical parameters obtained from experiments, for a primary components statistical analysis. As a result, the first approach to an acute stress reference scale has been proposed [8]. Proposed scale has been validated and proven to be robust measuring moderate psychological stress in healthy youngsters. Nevertheless, it is clear that proposed scale can't be widely used due to its inherent invasivity and high cost. To avoid these problems, we are elaborating a "workingcopy" of this reference scale based on electrophysiological signals.

\section{Expected and Unexpected Preliminary Results}

The recent experimental pilots already analyzedare providing data and specific information, some of them surprising,that will be used in the design of new experiments and in the design of new hardware and software tools to help in diagnostic and follow-up.

Some of them are the following:

A. A reference scale to quantitatively assess the stress level has been proposed and validated.

From the engineering point of view, this is a very important result that will allow faster advances based on the scientific communication using a reliable and repeatable measurement tool.

\section{B. Based on electrophysiological signals we have now a stress's multivariable biomarker}

Using a well-known set of physiological signals, the level of stress of an individual with a statistically significant correlation with biochemical markers and/or psychometric tests can be determined.

Using this multivariate biomarker, it is possible to discriminate between 5 stress levels even within the moderate stress category resulting from the use of TSST.

\section{Skin temperature seems to perfectly follow changes in the sympathetic-parasympathetic balance.}

Results found on the analysis of moderate stress in healthy young individuals show that temperature is changing in agreement with stressor stimulus with minimal delay times. Following this line, an infrared digital camera or a normal digital camera with appropriate filters could constitute a totally non-invasive first step in the stress analysis.

D. Glucose concentration in blood as well as a-amylaseand probablyglycatedhemoglobin could be taken as bio-markers of the chronic stress in adults.

Since for the measurement of blood glucose there are many devices today, this can be a chronic stress biomarker of widespreaduse.

An explanation could be found for evidence based on statistical studies that stress induces diabetes.

E. It is not demonstrated that Cytokines such as IL-6 and IL-10 are good biomarkers for chronic stress. 
J. Aguiló, Approaching Mental Disorders from the Engineering Point of View. Transactions on Machine Learning and Artificial Intelligence, Vol 5 No 4 August (2017); p: 833-841

Although some studies indicated that inflammation markers such as IL-6 and IL-10 may be markers of chronic stress, it has not been demonstrated in this study. Again, there are indications that point in this direction without being able to be taken as definitive.

\section{F. The effectiveness of treatments can be measured.}

The door to a tool to assess the quality of interventions using techniques of empowerment, mindfulness or biofeedback, drug based interventions or other forms of treatment is now opened.

\section{Related Challenges to Face}

\section{A. Fine-tuning the reference scale}

Only one stress scale? Only a single type of disorder under the stress name?

\section{B. Quantitative assessment of stress: a quality measurement of empowerment sessions.}

Results had and a proposal on what to do

C. Applying similar methodology to other mental disorders

Depression, Multiple Sclerosis, Epilepsy, Alzheimer, TDAH

D. Using developed scale and developed tools

The behavior under stress conditions as a biomarker for some disorders

E. Converging Technologies to remove the border along psychological and somatic responses?

Demonstrating quantitative associations between stress and diabetes, ictus, and heart attack between others

Could Technology and methodology set-up help in the early detection of anomalous behavior in young (including suicide) ?

\section{ACKNOWLEDGEMENTS}

This research line is supported by the CIBER-BBN(http://www.ciber-bbn.es)and for two of its consortium institutions: the Univ. Autónoma de Barcelona and the CSIC through the IMB-CNM (www.imbcnm.csic.es/). Direct funding is coming from MINECO (FIS-PI12/00514 and TIN2014-53567-R) and from IMI (https://www.imi.europa.eu/) which is public-private initiative under the framework of the Horizon 2020 the European research and innovation program.

\section{REFERENCES}

[1] B. M. Kudielka, D. H. Hellhammer, and C. Kirschbaum, "Ten years of research with the Trier Social Stress Test - revisited," in Social Neuroscience, 2007, pp. 56-83.

[2] K. L. Tamashiro, R. R. Sakai, C. A. Shively, I. N. Karatsoreos, and L. P. Reagan, "Chronic stress, metabolism, and metabolic syndrome.," Stress, vol. 14, no. 5, pp. 468-474, 2011. 
Transactions on Machine Learning and Artificial Intelligence Vol 5 No 4, Aug 2017

[3] Aguilo J, Ferrer-Salvans P, Garcia-Rozo A, et al. Project ES3: attempting to quantify and measure the level of stress. Rev Neurol. 2015;61(9):405-415.

[4] Arza A, Garzón JM, Hemando A, Aguiló J, Bailon R. Towards an objective measurement of emotional stress : Preliminary analysis based on heart rate variability. In: Medicine and Biology Society (EMBC), 2015 37th Annual International Conference of the IEEE. Milan, Italia, Agosto 2015.

[5] Hernando A, Lazaro J, Gil E, Arza A, Garzón-Rey JM et al. Inclusion of respiratory frequency information in Heart Rate Variability for a better analysis of Stress.IEEE JBHI2016; 20 (4): 1016-1025

[6] Knaus W, Zimmerman J. APACHE-acute physiology and chronic health evaluation: a physiologically based classification system. Crit care. 1981;9(8):591-7.

[7] Knaus WA, Draper EA, Wagner DP, Zimmerman JE. APACHE II: a severity of disease classification system. Crit Care Med. 1985;13(10):818-829.

[8] Jorge M. Garzón-Rey, Adriana Arza, Conchita de la Camara, Antonio Lobo, Antonio Armario, Jordi Aguilo. An Approach to an Acute Emotional Stress Reference Scale 